\title{
Low refractive index Si nanopillars on Si substrate
}

\author{
Gong-Ru Lin ${ }^{\mathrm{a}}$ \\ Graduate Institute of Electro-Optical and Department of Electrical Engineering, National Taiwan \\ University, No. 1, Roosevelt Road Sec. 4, Taipei 106, Taiwan, Republic of China
}

Ya-Chung Chang and En-Shao Liu

Research Center for Applied Sciences, Academia Sinica, 128 Academia Rd., Sec. 2 Nankang, Taipei 115, Taiwan, Republic of China

Hao-Chung Kuo and Huang-Shen Lin

Department of Photonics, National Chiao Tung University, 1001, Ta Hsueh Road, Hsinchu 300, Taiwan Republic of China

(Received 14 December 2006; accepted 11 April 2007; published online 3 May 2007)

\begin{abstract}
Low refractive index of a stalagmitelike high-aspect-ratio Si nanopillar array on Si dry etched using a self-aggregated Ni nanodot mask is demonstrated, which exhibits two minimum reflectances of 1.23 and 1.4 with corresponding refractive indices of 1.25 and 1.48 at 400 and $1200 \mathrm{~nm}$, respectively. Angular dependency of the transverse electrical mode reflectance at $632.8 \mathrm{~nm}$ of the surface roughened Si nanopillar array is released, and the Brewster angle of $63^{\circ}$ with equivalent refractive index of 1.5 is determined. (C) 2007 American Institute of Physics.
\end{abstract}

[DOI: $10.1063 / 1.2736281]$

Versatile nanoscale silicon $(\mathrm{Si})$ or silica structures with extraordinary electrical and optical characteristics have recently been synthesized and investigated, such as porous $\mathrm{Si}^{1}$ or nanoporous silica, ${ }^{2} \mathrm{Si}$ nanopyramid, ${ }^{3} \mathrm{Si}$ nanopillar, ${ }^{4,5}$ or silica nanorod $^{6,7}$ array on Si substrate. These surface roughened nanostructures are considered as promising systems for developing electronic or photonic devices. Enhanced optical phonon response, variable selection rules, and a lowfrequency shift of $0.5 \mathrm{~cm}^{-1}$ of Raman band corresponding to the quantum size effect in Si nanorods were observed. ${ }^{8}$ In particular, the modified optical refractive index of the aforementioned $\mathrm{Si}$ or silica nanostructures is of great interest, which plays an important role on the performance of $\mathrm{Si}$ based photonic devices involving high-reflection or antireflection multilayer coating, resonant cavity, and photonic crystal structures. ${ }^{6,7}$ Another demand for potential application in Si based photovoltaic solar cells also becomes a driving force for emerging Si or silica nanostructures of ultralow refractive indices. Not long ago, the silica nanorod array on $\mathrm{Si}$ substrate with unprecedented refractive index of $1.08{ }^{7}$ even smaller than those of the $\mathrm{MgF}_{2}, \mathrm{CaF}_{2}$, and $\mathrm{SiO}_{2}$ films used for depositing antireflection coatings, were demonstrated in visible spectral range. Nonetheless, the study on reflectance or refractive index spectroscopy of a similar $\mathrm{Si}$ nanopillar structure was seldom addressed. In this work, we investigate the optical reflectance spectroscopy of a Si nanopillar array fabricated using a $\mathrm{Ni}$ nanodot mask assistant reactive-ion-etching process. Ultralow refractive index in the UV-visible-near infrared wavelength range in comparison with other dielectric materials is demonstrated and elucidated.

$\mathrm{A} \mathrm{Ni} / \mathrm{SiO}_{2}$ nanomask assistant dry-etching procedure is employed to fabricate the Si nanopillars on Si substrate, as schematically shown in Fig. 1. A 50-nm-thick Ni film is evaporated on the $\mathrm{SiO}_{2} / \mathrm{Si}$ substrate using an e-beam evapo-

\footnotetext{
a) Author to whom correspondence should be addressed; electronic mail: grlin@ntu.edu.tw
}

rating system with $\mathrm{Ni}$ deposition rate of $0.1 \AA$ /s under an applied current of $70 \mathrm{~mA}$. The $\mathrm{SiO}_{2}$ buffered layer with a thickness of $200 \AA$ is deposited by using a plasma enhanced chemical vapor deposition under standard recipe. Subsequently, a rapid thermal annealing process at $850{ }^{\circ} \mathrm{C}$ for $22 \mathrm{~s}$ under the $\mathrm{N}_{2}$ flowing gas of 5 SCCM (SCCM denoted cubic centimeter per minute of STP) is performed to self-aggregate the $\mathrm{Ni}$ nanodots on $\mathrm{SiO}_{2} / \mathrm{Si}$ substrate. By using the $\mathrm{Ni}$ nanodots with area density of $5 \times 10^{11} \mathrm{~cm}^{-2}$ as a nanomask, the $\mathrm{Si}$ substrate is dry etched in a planar-type inductively coupled plasma-reactive ion etching (ICP-RIE) system (SAMCO ICP-RIE 101iPH) at rf frequency of $13.56 \mathrm{MHz}$ and the ICP/bias power conditions of 100/50 Watts. The etching gases of $\mathrm{CF}_{4}$ and $\mathrm{Ar}$ mixture with a condition of $\mathrm{CF}_{4} / \mathrm{Ar}=40 / 40 \mathrm{SCCM}$ were introduced into the reactive chamber through individual electronic mass flow controllers. The chamber pressure of $0.66 \mathrm{~Pa}$ remains unchanged during an etching duration of 5-7 min. To avoid the gradual oxidation effect, the Si sample is dipped in methyl alcohol before diagnosis. The sample is dried with a 2 min nitrogen purge prior to the measurement. The surface reflectance of the $\mathrm{Si}$ nanopillars on $\mathrm{Si}$ substrate is characterized at wavelength

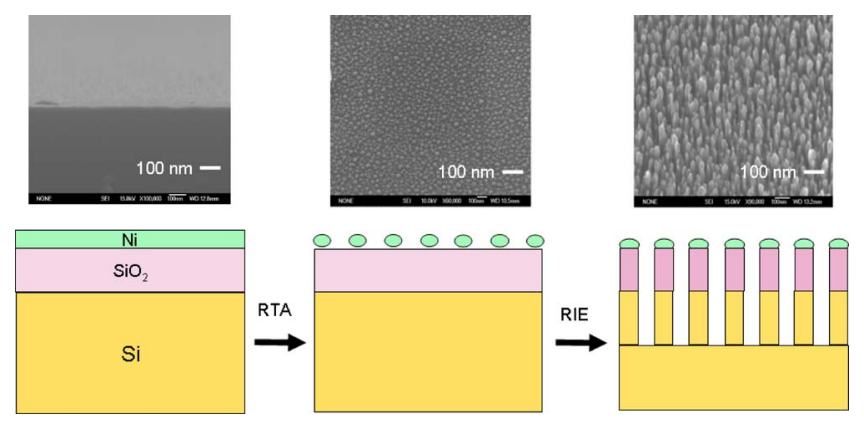

FIG. 1. (Color online) Schematic diagram for fabricating Si nanopillar array on $\mathrm{Si}$ using a self-assembled Ni nanodot etching mask on $\mathrm{SiO}_{2} / \mathrm{Si}$ substrate is illustrated. The scanning electron microscopic (SEM) photographs of unprocessed $\mathrm{Si}$ substrate, self-aggregated $\mathrm{Ni}$ on $\mathrm{SiO}_{2} / \mathrm{Si}$, and ICP-RIE etched $\mathrm{Si}$ nanopillars on $\mathrm{Si}$ are shown for example. 


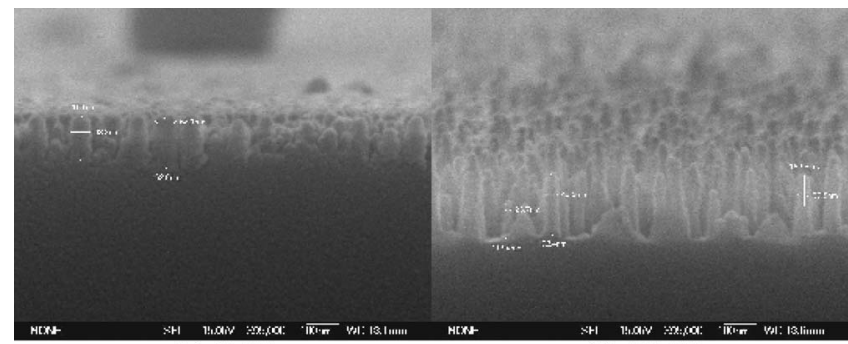

(a) $50 \mathrm{~W}$

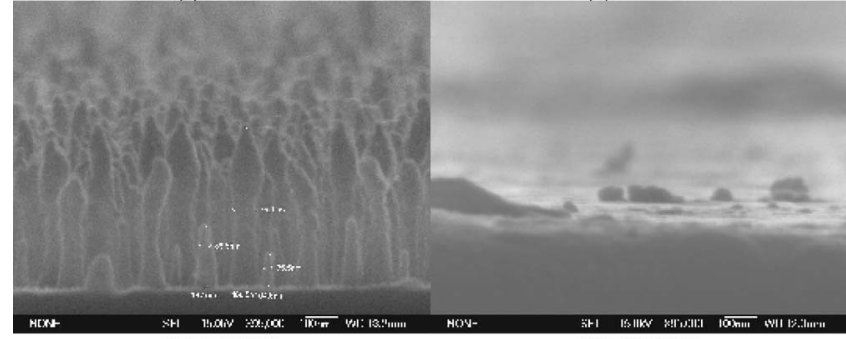

(c) $150 \mathrm{~W}$

(d) $200 \mathrm{~W}$

FIG. 2. SEM photographs of Si nanopillar array obtained by detuning the ICP power at (a) 50, (b) 100, (c) 150, and (d) $200 \mathrm{~W}$.

ranging from 300 to $1700 \mathrm{~nm}$. After removing the $\mathrm{Ni} / \mathrm{SiO}_{2}$ nanomask, the $\mathrm{Si}$ nanopillars with a perpendicular sidewall and high aspect ratio are obtained, as shown in Fig. 1. The density, diameter, and height of $\mathrm{Si}$ nanopillars are tunable from $6.5 \times 10^{10}$ to $5 \times 10^{11} \mathrm{~cm}^{-2}$, from 30 to $70 \mathrm{~nm}$, and from 100 to $480 \mathrm{~nm}$, respectively. Figure 2 clearly shows that the ICP power of the ICP-RIE strongly affects the etching rate and substrate selectivity. At an ICP power of $50 \mathrm{~W}$, the etching selectivity is high but the etching rate is very low, leading to the incompletely formatted $\mathrm{Si}$ nanopillars with average height of $100 \mathrm{~nm}$ and extremely low aspect ratio of $<2$ shown in Fig. 2(a). Increasing ICP power to $100 \mathrm{~W}$ results in a compromise between substrate selectivity and etching rate, providing a higher Si nanopillar $(>180 \mathrm{~nm})$ with smaller size $(<35 \mathrm{~nm})$ and enhanced aspect ratio of 5 . Such a trade-off between the etching rate and the aspect ratio becomes less significant at ICP power ranging between 100 and $150 \mathrm{~W}$. The etching depth reaches its maximum at ICP power of $150 \mathrm{~W}$, providing a $\mathrm{Si}$ pillar array as high as $480 \mathrm{~nm}$ with its aspect ratio ranging from 5 to 8 , as shown in Fig. 2(c). Afterwards, Fig. 2(d) illustrates that the Si nanopillars were etched out due to the low etching selectivity and high etching rate at ICP power higher than $200 \mathrm{~W}$.

The Si nanopillar array with highest aspect ratio was selected to characterize its optical reflectance spectroscopy and to compare with that of bulk $\mathrm{Si}$ substrate, as shown in Fig. 3. As a result, the reflectance of Si wafer decreases after nanomask formation and nanopillar etching. It is observed that the Si nanopillar array on $\mathrm{Si}$ substrate has a maximum reflectance $<8.6 \%$ (at $1013 \mathrm{~nm}$ ), corresponding to a refractive index of $<1.82$ at wavelength ranging from 320 to $1650 \mathrm{~nm}$. However, it is already much smaller, less than that of the unprocessed $\mathrm{Si}$ substrate (see Fig. 3). In comparison, the standard silica coating exhibits a reflectance of 5.2\% and a refractive index around 1.4 within the wavelength region. In the visible spectral range, $\mathrm{MgF}_{2}, \mathrm{CaF}_{2}$, and $\mathrm{SiO}_{2}$ with refractive indices of $1.39,1.44$, and 1.46 , respectively, are currently available candidates with lowest refractive indices. ${ }^{6,7}$ In particular, the Si nanopillar array has ultralow refractive indices of 1.23 and 1.4 at two wavelength Downloaded 10 Feb 2009 to 140.112 .113 .225 . Redistribution subject

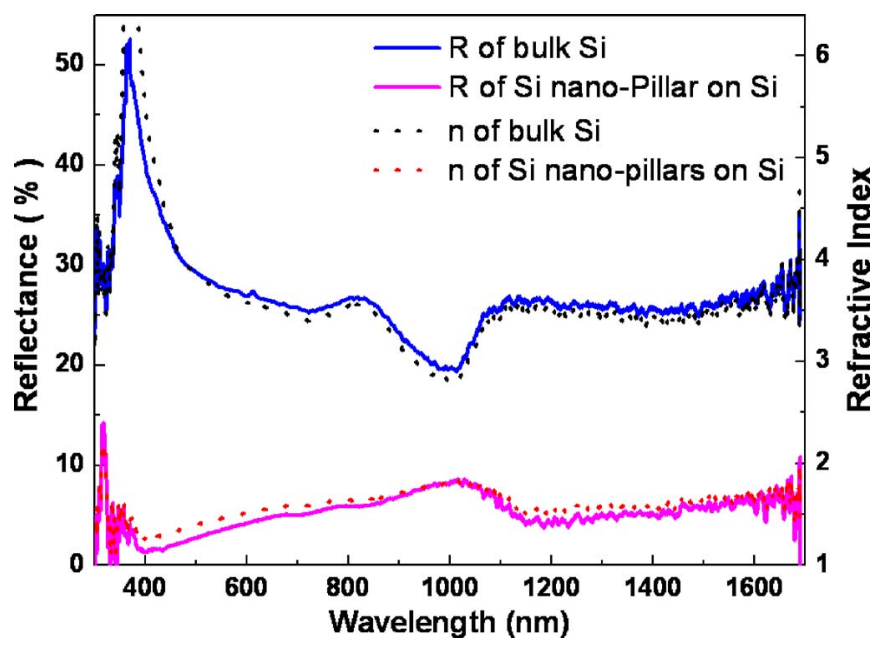

FIG. 3. (Color online) Surface reflectance and corresponding refractive indices of unprocessed bulk Si and Si nanopillar array on Si substrate.

regions around 400 and $1200 \mathrm{~nm}$, respectively. The Si nanopillar array roughened surface inevitably causes a reflectance lower than that of the Si substrate on account of its highly roughened surface. ${ }^{7}$ We also experimentally found that the $\mathrm{Si}$ nanopillars encapsulated with $\mathrm{Ni}$ and $\mathrm{SiO}_{2}$ nanomask exhibit even lower reflectance than the pure Si nanopillar array due to the existence of oxide nanodots. In contrast to the traditional antireflection coatings for Si-based photon energy transforming devices using optically homogeneous materials with a refractive index lower than that of a cell semiconductor, such a low-reflectance Si nanopillar array further motivates the development of $\mathrm{Si}$ nanomaterials which benefits from fully processing compatibility with the Si based photovoltaic solar cell. On the other hand, the emergence of the nanostructural materials also provides flexibility on gradually detuning the refractive index between 1.2 in vacuum and 3.9 in bulk $\mathrm{Si}$ at visible wavelengths. Recently, an optical film consisting of $\mathrm{SiO}_{2}$ nanorod array have been demonstrated to show ultralow refractive index of 1.08 with a film thickness of $1.35 \mu \mathrm{m},{ }^{7}$ in which the angled $\mathrm{SiO}_{2}$ nanorods with both the gap spacing and diameter are much smaller than the wavelength of visible light to minimize Mie and Rayleigh scattering. Moreover, similar antireflection phenomenon was also found on the porous Si surface, revealing a reduced refractive index of $1.75-2.15 .^{1}$ In our case, a refractive index of 1.23 at $400 \mathrm{~nm}$ is obtained in the Si nanopillar array on Si substrate with an average height of only $450 \mathrm{~nm}$.

To realize the antireflection property of the Si nanopillar array on the bulk Si substrate for potential application on photovoltaic solar cells, we further characterize the angular dependent reflectance by using TE and TM polarized beams of a HeNe laser at $632.8 \mathrm{~nm}$. The angular dependent reflectance at different incident polarizations in Fig. 4 shows similar low-reflectance phenomenon with that of the silica nanopillar array observed by Xi et al. ${ }^{7}$ previously. The Brewster angle of the bulk Si related to a minimum reflectance of $8.21 \times 10^{-2}$ for the TE-mode laser beam is determined as $76^{\circ}$. Possible mechanism of the slightly deviated Brewster angle as well as refractive index measured from the unprocessed Si wafer is mainly attributed to the contribution of the surface oxide layer naturally grown on the unprocessed $\mathrm{Si}$ substrate. In contrast, the Si nanopillar array exhibits exto AIP license or copyright; see http://apl.aip.org/apl/copyright.jsp 


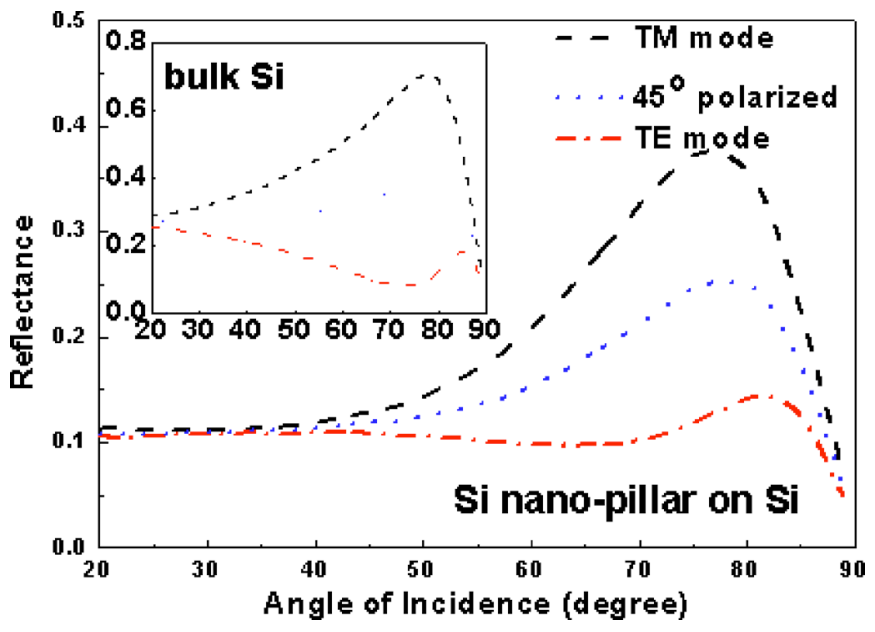

FIG. 4. (Color online) Angular dependent reflectance of Si nanopillar array and bulk Si substrate (inset) measured by HeNe laser at $632.8 \mathrm{~nm}$ and different polarizations.

tremely low TE-mode reflectance of $<1 \times 10^{-1}$ associated with a change in reflectance of only 0.016 at incident angles $<75^{\circ}$. With the formula of $\theta_{\text {Brewster }}=\tan ^{-1}\left(n_{\mathrm{si}} / n_{\text {air }}\right)$, the determined Brewster angle with a minimum reflectance of $9.05 \times 10^{-2}$ is about $63^{\circ}$, corresponding to an equivalent refractive index of only 1.5 at wavelength of $632.8 \mathrm{~nm}$ for such a high-aspect-ratio Si nanopillars on Si substrate. The calculated refractive index is relatively in good agreement with that obtained from the reflectance spectroscopy (see Fig. 3). Another direct evidence to support the ultralow refractive index of the Si nanopillar array is its greatly reduced reflectance at lower incident angles as compared to the unprocessed bulk Si substrate. For example, the reflectance of $\mathrm{Si}$ substrate at an incident angle of $20^{\circ}$ is significantly reduced from $29.5 \%$ to $10.5 \%$ after formatting Si nanopillars, corresponding to a refractive index changing from 3.378 to 1.958. Identical trend can also be obtained under both the $45^{\circ}$-polarized and TE-mode incident conditions. The more important observation is that the Si nanopillar array exhibits a less angle-dependent TE-mode reflectance in comparison with the bulk Si substrate, which indicates that the polarization dependence of reflectance has been released by introduc- ing such a surface roughened $\mathrm{Si}$ nanopillar array on $\mathrm{Si}$ substrate.

In conclusion, we have investigated the reflectance spectroscopy of Si nanopillar array on Si substrate with high aspect ratio. The stalagmitelike $\mathrm{Si}$ nanopillar array is fabricated by dry etching the $\mathrm{SiO}_{2} / \mathrm{Si}$ substrate with selfaggregated Ni nanodot array based etching mask. The density, diameter, and height of Si nanopillars are tunable from $6.5 \times 10^{10}$ to $5 \times 10^{11} \mathrm{~cm}^{-2}$, from 30 to $70 \mathrm{~nm}$, and from 100 to $480 \mathrm{~nm}$, respectively. The Si nanopillar array inevitably causes a reflectance lower than that of the Si substrate on account of its air/Si mixed structure and highly roughened surface, leading to an ultralow reflectance of $<8.6 \%$ (at $1013 \mathrm{~nm}$ ) and corresponding refractive index of $<1.82$ at wavelength ranging from 320 to $1650 \mathrm{~nm}$. A minimum reflectance and refractive index of 1.23 and 1.25 at wavelength of $400 \mathrm{~nm}$ is obtained for the Si nanopillar array on Si with average height of $450 \mathrm{~nm}$ and aspect ratio of 5. The Brewster angle of $63^{\circ}$ obtained by TE-mode angular dependent reflectance at $632.8 \mathrm{~nm}$ gives rise to an equivalent refractive index of nearly 1.5 , which is relatively in good agreement with the reflectance spectroscopic analysis. The angular reflectance further reveals a released polarization dependency of such a surface roughened Si nanopillar array on Si substrate.

This work was supported in part by the National Science Council, Taiwan, Republic of China under Grant No. NSC95-2221-E-002-448.

${ }^{1}$ E. Chambon, E. Florentin, T. Torchynska, J. Gonzalez-Hernandez, and Y. Vorobiev, Microelectron. J. 36, 514 (2005).

${ }^{2}$ G. Wu, J. Wang, J. Shen, T. Yang, Q. Zhang, B. Zhou, Z. Deng, B. Fan, D. Zhou, and F. Zhang, Mater. Sci. Eng., B 78, 135 (2000).

${ }^{3}$ G.-R. Lin, H.-C. Kuo, H.-S. Lin, and C.-C. Kao, Appl. Phys. Lett. 89, 073108 (2006).

${ }^{4}$ T. Tada, A. Hamoudi, T. Kanayama, and K. Koga, Appl. Phys. Lett. 70, 2538 (1997).

${ }^{5}$ T. Tada and T. Kanayama, J. Vac. Sci. Technol. B 16, 3934 (1998).

${ }^{6}$ S.-Y. Lin, E. Chow, V. Hietala, P. R. Villeneuve, and J. D. Joannopoulos, Science 282, 274 (1998).

${ }^{7}$ J.-Q. Xi, J. K. Kim, and E. F. Schubert, Nano Lett. 5, 1385 (2005).

${ }^{8}$ V. V. Poborchii, T. Tada, and T. Kanayama, Physica E (Amsterdam) 7, 545 (2000). 